\title{
On Non-real Eigenvalues of Schrödinger Operators in a Weighted Hilbert Space
}

\author{
Dedicated to Professor Teruo Ikebe on his sixtieth birthday
}

By

Masaharu Arai* and Osanobu YAMADA*

\section{$\S 1$. Introduction}

In this paper we are concerned with the Schrödinger operator

$$
L=-\sum_{j, k=1}^{n}\left(\frac{\partial}{\partial x_{j}}+i b_{j}(x)\right) a_{j k}(x)\left(\frac{\partial}{\partial x_{k}}+i b_{k}(x)\right)+V(x),
$$

where $i=\sqrt{-1}$ and $b_{j}(x), V(x)$ are real valued functions. For simplicity, we consider only the case $a_{j k}(x) \equiv \delta_{j k}$ in the present section. Our aim is to study the existence or the non-existence of eigenfunctions $u(x)$ of $L$ such that

$$
\begin{aligned}
& L u=z u, \\
& u \in H_{2, l o c}, \quad\left(1+|x|^{2}\right)^{-s / 2} u(x) \in L^{2}\left(\boldsymbol{R}^{n}\right)
\end{aligned}
$$

for a non-real number $z$ and $s \geq 0$. Under our conditions stated in $\S 2$ the non-existence of non-trivial solutions of $u(x)$ satisfying (1.2) is equivalent to the following Proposition (P);

(P) the set $\left\{(L-\bar{z}) u \mid u \in C_{0}^{\infty}\left(\mathbb{R}^{n}\right)\right\}$ is dense in the weighted space

$$
L_{s}^{2}=\left\{f \mid\left(1+|x|^{2}\right)^{s / 2} f \in L^{2}\left(\boldsymbol{R}^{n}\right)\right\}
$$

(see Remark 2.2 in $\S 2$ ), where $\bar{z}$ is the complex conjugate of $z$. It is well-known that the Proposition (P) with $s=0$ and $z= \pm \sqrt{-1}$ is equivalent

Communicated by S. Matsuura, June 11, 1990.

1991 Mathematics Subject Classification: 35P15

* Department of Mathematics, Ritsumeikan University, Noji-cho, Kusatsu, Shiga 525, Japan 
to the essential self-adjointness of the symmetric operator $L$ restricted to $C_{0}^{\infty}\left(\boldsymbol{R}^{n}\right)$, which is the case under our condition so that the equation (1.2) with $s \leq 0$ has no non-trivial solutions. But the problem with $s>0$ is not trivial.

Ikebe-Saitō [2] proves the Proposition (P) in order to study the limiting absorption method for Schrödinger operators with short range potentials. The second writer [5] treats exploding potentials, i.e., those satisfying the Stummel condition and

$$
\begin{gathered}
V(x) \rightarrow-\infty \quad \text { as } \quad r=|x| \rightarrow \infty, \\
V(x)=O\left(r^{\alpha}\right) \quad \text { as } \quad r \rightarrow \infty,
\end{gathered}
$$

for some $\alpha<2$ and shows (P) and the limiting absorption principle. But he has encountered a difficulty to prove $(\mathrm{P})$ for $\alpha=2$ in (1.3)

We note here that the non-existence of eigenfunctions of (1.2) is also shown by Simon [4], Theorem 2.2 for a class of potentials, roughly speaking, bounded from below.

Our interest in the present paper is to investigate the case that $V(x)$ satisfies

$$
V(x) \geq-C|x|^{2},|x| \geq R
$$

for some positive $C$ and $R$. We shall show the non-existence of eigenfunctions of (1.2) under the condition

$$
|\operatorname{Im} z|>2 s \sqrt{C}
$$

where $\operatorname{Im} z$ is the imaginary part of $z$. This condition (1.4) is optimal in the sense stated in $\S 4$.

Our assertion will be stated in $\S 2$ and will be proved in $\S 3$. The optimality of the condition (1.4) will be shown in $\S 4$ by giving some examples. We shall consider these problems not only for $\left[a_{j k}(x)\right] \equiv I$ (identity matrix) but also for positive symmetric matrices $\left[a_{j k}(x)\right]$ for $x \in \mathbb{R}^{n}$.

\section{§2. Assumptions and Results}

The following condition (A) on $L$ of (1.1) is assumed throughout this paper: 
(A-1) The $n \times n$ matrices $A(x)=\left[a_{j k}(x)\right]$ are real symmetric and positive definite for $x \in \boldsymbol{R}^{n}$ with $C^{2}$ components.

(A-2) Let $a^{+}(x)$ denote the greatest eigenvalue of $A(x)$ and put

$$
a^{*}(r)=\max _{|x|=r} a^{+}(x)
$$

Then

$$
\begin{gathered}
\rho(r) \equiv \int_{0}^{r} \frac{d s}{\sqrt{a^{*}(s)}} \rightarrow \infty, \quad r \rightarrow \infty . \\
\left(\text { If } a_{j k}(x) \equiv \delta_{j k}, a^{+}(x)=a^{*}(r) \equiv 1 \text { and } \rho(r)=r .\right)
\end{gathered}
$$

(A-3) Each component $b_{j}(x)$ of the vector potential is a real valued $C^{1}$ function.

(A-4) The potential $V(x)$ is real valued and can be decomposed into $V(x)=V_{1}(x)+V_{2}(x)$ as follows;

i) $V_{1}(x)$ satisffes

$$
V_{1}(x) \geq-C_{1} \rho(|x|)^{2}-C_{2} \quad\left(x \in \boldsymbol{R}^{n}\right)
$$

for some positive constants $C_{1}$ and $C_{2}$, and belongs to the Stummel class $Q_{\alpha, l o c}$ for a positive number $\alpha<1$ i.e.,

$$
M\left[V_{1}\right](x) \equiv\left[\int_{|x-y| \leq 1} \frac{\left|V_{1}(y)\right|^{2}}{|x-y|^{n-4+\alpha}} d y\right]^{1 / 2}
$$

is a locally bounded function of $x \in R^{n}$.

ii) $V_{2}(x)$ also belongs to $Q_{\alpha, l o c}$ and satisfies the following conditions (A-5) and (A-6).

(A-5) Let $a^{-}(x)$ be the least eigenvalue of $A(x)$ and let $V^{-}(x)=\max (-V(x)$, $0)$. Then, there exists a positive constant $C_{3}$ such that

$$
M\left[V_{2}^{-}\right](x) \leq C_{3} a^{-}(x) \quad\left(x \in R^{n}\right) .
$$

(A-6) $M\left[V_{2}^{-}\right](x)=o\left(\rho(r)^{2}\right), r=|x| \rightarrow \infty$.

For a real number $s$ and the function $\rho(r)$ above, $L_{s, \rho}^{2}$ denotes the weighted Hilbert space consisting of all functions $f(x)$ satisfying

$$
\|f\|_{s, \rho}=\left(\int_{\mathbb{R}^{n}}\left[1+\rho(r)^{2}\right]^{s}|f(x)|^{2} d x\right)^{1 / 2}<\infty
$$


with the inner product

$$
(f, g)_{s, \rho}=\int_{\mathbb{R}^{n}}\left[1+\rho(r)^{2}\right]^{s} f(x) \overline{g(x)} d x
$$

When $\rho(r)=r, L_{s, \rho}^{2}$ is written simply as $L_{s}^{2} . \quad L_{0, \rho}^{2}=L_{0}^{2}$ is the usual Hilbert space $L^{2}\left(\mathbb{R}^{n}\right)$.

$H_{2}=H_{2}\left(\mathbb{R}^{n}\right)$ is the Sobolev space of all functions $u \in L^{2}\left(\mathbb{R}^{n}\right)$ such that $\Delta u \in L^{2}\left(\mathbb{R}^{n}\right) . \quad H_{2, l o c}$ is the class of all locally $H_{2}$ functions.

Theorem 2.1. Assume the condition $(A)$ and let $z \in \mathbb{C}$ and $s \geq 0$ satisfy

$$
\operatorname{Im} z \mid>2 s \sqrt{C_{1}}
$$

Then, there exist no non-trivial solutions $u \in L_{-s, \rho}^{2} \cap H_{2, l o c}$ satisfying

$$
L u=z u \text {. }
$$

This theorem will be proved in the next section.

Remark 2.2. Under the condition (A), the set

$$
(L-\bar{z}) C_{0}^{\infty}=\left\{(L-\bar{z}) f \mid f \in C_{0}^{\infty}\left(\boldsymbol{R}^{n}\right)\right\}
$$

is dense in $L_{s, \rho}^{2}$ for non-real $z$, if and only if (2.2) has no non-trivial solutions in $L_{-s . \rho}^{2} \cap H_{2, l o c}$. This fact can be obtained by the same argument as in Ikebe-Saitō [2], Lemma 1.10.

Remark 2.3 If $V_{1}(x)$ satisfies

$$
V_{1}(x)=o\left(\rho(r)^{2}\right), \quad r \rightarrow \infty
$$

then the constant $C_{1}$ in (A.4) can be taken arbitrarily small, and the assumption (2.1) of Theorem 2.1 is valid for every non-real $z$.

\section{§3. Proof of Theorem 2.1}

We start with the following Lemma. 
Lemma 3.1. Let $f(x)$ and $g(x)$ be real valued $C^{1}$-functions on $\boldsymbol{R}^{n}$ and $u(x)$ be an $H_{2, l o c}$ function. Assume that $f(x)$ has compact support. Then we have

$$
\begin{aligned}
\int_{\boldsymbol{R}^{n}} f^{2} g^{2}(L u) \bar{u} d x= & \int_{\boldsymbol{R}^{n}}\left\{f^{2} g^{2}\left[\langle A D u, \overline{D u}\rangle+\left(V_{1}+V_{2}\right)|u|^{2}\right]\right. \\
& +2 f g[f\langle A \nabla g, D u\rangle+g\langle A \nabla f, D u\rangle] \bar{u}\} d x,
\end{aligned}
$$

where

$$
\begin{aligned}
\langle\vec{a}, \vec{b}\rangle & =\sum_{j=1}^{n} a_{j} b_{j}, \quad\langle A \vec{a}, \vec{b}\rangle=\sum_{j, k=1}^{n} a_{j k}(x) a_{k} b_{j} \\
\text { for } & \vec{a}=\left(a_{1}, a_{2}, \cdots, a_{n}\right), \quad \vec{b}=\left(b_{1}, b_{2}, \cdots, b_{n}\right), \\
\nabla & =\left(\frac{\partial}{\partial x_{1}}, \frac{\partial}{\partial x_{2}}, \cdots, \frac{\partial}{\partial x_{n}}\right) \\
D & =\left(D_{1}, D_{2}, \cdots, D_{n}\right), \quad D_{j}=\frac{\partial}{\partial x_{j}}+i b_{j}(x) \\
\overline{D u} & =\left(\overline{D_{1} u}, \overline{D_{2} u}, \cdots, \overline{D_{n} u}\right) .
\end{aligned}
$$

The above Lemma can be proved directly by integration by parts.

Lemma 3.2. Assume the condition (A), and let $z \in C, s \geq 0$ and $u \in L_{-s, \rho}^{2} \cap H_{2, l o c}$ be a solution of (2.2). Then, for any $C_{1}^{\prime}>C_{1}$ there exists a positive constant $m_{0}$ such that

$$
\int_{\boldsymbol{R}^{n}}\left(m_{0}+\rho(r)^{2}\right)^{-s-1}\langle A D u, \overline{D u}\rangle d x \leq C_{1}^{\prime} \int_{\mathbb{R}^{n}}\left(m_{0}+\rho(r)^{2}\right)^{-s}|u|^{2} d x
$$

Proof. Take a $C^{\infty}$ function $\varphi$ on $\boldsymbol{R}$ such that

$$
\varphi(t)=1(t \leq 1), \varphi(t)=0(t \geq 2) \quad \text { and } \quad 0 \leq \varphi(t) \leq 1(1 \leq t \leq 2)
$$

and put

$$
f_{R}(x)=\varphi(\rho(|x|)-R+1) .
$$


Making use of Lemma 3.1 with $f=f_{R}$ and

$$
g=\left(m+\rho(r)^{2}\right)^{-(s+1) / 2} \quad(m>0)
$$

for the integral

$$
\int_{\mathbb{R}^{n}} f_{R}^{2} g^{2}(L u) \bar{u} d x=z \int_{\mathbb{R}^{n}} f_{R}^{2} g^{2}|u|^{2} d x
$$

and taking the real part, we have

$$
\begin{aligned}
\int_{\mathbb{R}^{n}}( & \left.m+\rho(r)^{2}\right)^{-s-1} f_{R}^{2}\langle A D u, \overline{D u}\rangle d x \\
= & -\int_{\mathbb{R}^{n}}\left\{f_{R}^{2} g^{2}\left(V_{1}+V_{2}-\operatorname{Re} z\right)|u|^{2}\right. \\
& \left.+2 f_{R}^{2} g \operatorname{Re}[\langle A \nabla g, D u\rangle \bar{u}]+2 f_{R} g^{2} \operatorname{Re}\left[\left\langle A \nabla f_{R}, D u\right\rangle \bar{u}\right]\right\} d x \\
\leq & \int_{\mathbb{R}^{n}} f_{R}^{2} g^{2}\left(-V_{1}+|\operatorname{Re} z|\right)|u|^{2} d x+\int_{\mathbb{R}^{n}} f_{R}^{2} g^{2} V_{2}^{-}|u|^{2} d x \\
& +\int_{\mathbb{R}^{n}} f_{R}^{2} g^{2}|u|^{2} d x+\int_{\mathbb{R}^{n}} f_{R}^{2}|\langle A \nabla g, D u\rangle|^{2} d x \\
& +\left.\eta \int_{\mathbb{R}^{n}} f_{R}^{2} g^{2}\left|\left\langle A \nabla f_{R}, D u\right\rangle^{2} d x+\frac{1}{\eta} \int_{\mathbb{R}^{n}} g^{2}\right| u\right|^{2} d x \\
\equiv & I_{1}+I_{2}+I_{3}+I_{4}+I_{5}+I_{6}
\end{aligned}
$$

for any $\eta>0$. We shall estimate $I_{1} \sim I_{6}$ as follows.

The condition (A.4) gives

$$
\begin{aligned}
& I_{1}=\int_{\mathbb{R}^{n}} f_{R}^{2} g^{2}\left(-V_{1}+|\operatorname{Re} z|\right)|u|^{2} d x \\
& \leq \int_{\mathbb{R}^{n}}\left(m+\rho(r)^{2}\right)^{-s-1}\left(C_{1} \rho(r)^{2}+C_{2}+|\operatorname{Re} z|\right)|u|^{2} d x \\
& \leq\left(C_{1}+\frac{C_{2}^{\prime}}{m}\right) \int_{\mathbb{R}^{n}}\left(m+\rho(r)^{2}\right)^{-s}|u|^{2} d x
\end{aligned}
$$


where we put $C_{2}^{\prime}=C_{2}+|\operatorname{Re} z|$. Ikebe-Kato [1], Lemma 2 and (A.4) yield

$$
\begin{aligned}
I_{2} & =\int_{\mathbb{R}^{n}} f_{R}^{2} g^{2} V_{2}^{-}(x)|u|^{2} d x \\
& \leq C_{0} \eta^{\alpha / 2} \int_{\boldsymbol{R}^{n}} M\left[V_{2}^{-}\right]\left\{f_{R}^{2} g^{2}|D u|^{2}+\left|\nabla\left(f_{R} g\right)\right|^{2}|u|^{2}+\eta^{-2} f_{R}^{2} g^{2}|u|^{2}\right\} d x
\end{aligned}
$$

for any $0<\eta<1$ and some positive constant $C_{0}$ independent of $\eta$ and $R$ (see also Jörgens [3], §3). The above inequality (3.5), the condition (A-5), and

$$
M\left[V_{2}^{-}\right](x) \leq \eta^{2} \rho(r)^{2}+C(\eta)
$$

for any $\eta>0$ and some constant $C(\eta)>0$, which is a consequence of (A-6), imply

$$
\begin{aligned}
I_{2} & \leq C_{0} \eta^{\alpha / 2} \int_{R^{n}}\left\{C_{3} f_{R}^{2} g^{2}\langle A D u, \overline{D u}\rangle+2 C_{3} f_{R}^{2}\langle A \nabla g, \nabla g\rangle|u|^{2}\right. \\
& \left.+2 C_{3} g^{2}\left\langle A \nabla f_{R}, \nabla f_{R}\right\rangle|u|^{2}+\rho(r)^{2} f_{R}^{2} g^{2}|u|^{2}+\frac{C(\eta)}{\eta^{2}} f_{R}^{2} g^{2}|u|^{2}\right\} d x
\end{aligned}
$$

for any $0<\eta<1$. In view of the definition (3.2) of $f_{R}$ we have

$$
\begin{aligned}
&\left\langle A \nabla f_{R}, \nabla f_{R}\right\rangle=\langle A \hat{x}, \hat{x}\rangle \rho^{\prime}(r)^{2} \varphi^{\prime}(\rho(r)-R+1)^{2} \\
&=\langle A \hat{x}, \hat{x}\rangle a^{*}(r)^{-1} \varphi^{\prime}(\rho(r)-R+1)^{2} \\
& \leq \varphi^{\prime}(\rho(r)-R+1)^{2} \leq C_{4} \\
&(\hat{x}=x /|x|)
\end{aligned}
$$

for a positive constant $C_{4}$, and similarly

$$
\begin{aligned}
\langle A \nabla g, \nabla g\rangle & =\langle A \hat{x}, \hat{x}\rangle a^{*}(r)^{-1}(s+1)^{2} \rho(r)^{2}\left(m+\rho(r)^{2}\right)^{-s-3} \\
& \leq(s+1)^{2}\left(m+\rho(r)^{2}\right)^{-s-2}
\end{aligned}
$$




$$
\begin{aligned}
& \leq \frac{(s+1)^{2}}{m}\left(m+\rho(r)^{2}\right)^{-s-1} \\
& \leq \frac{(s+1)^{2}}{m^{2}}\left(m+\rho(r)^{2}\right)^{-s}
\end{aligned}
$$

for any $m>0$. Gathering (3.6), (3.7) and (3.8) gives

$$
\begin{aligned}
I_{2} \leq & C_{0} \eta^{\alpha / 2} \int_{\mathbb{R}^{n}}\left(m+\rho(r)^{2}\right)^{-s}\left[C_{3} f_{R}^{2}\left(m+\rho(r)^{2}\right)^{-1}\langle A D u, \overline{D u}\rangle\right. \\
& \left.+2 C_{3}\left(\frac{s+1}{m}\right)^{2}|u|^{2}+\frac{2 C_{3} C_{4}}{m}|u|^{2}+|u|^{2}+\frac{C(\eta)}{m \eta^{2}}|u|^{2}\right] d x
\end{aligned}
$$

for any positive $m$ and $\eta<1$. For $I_{3}$ and $I_{6}$ we have

$$
\begin{aligned}
I_{3}+I_{6} & =\int_{\mathbb{R}^{n}}\left\{f_{R}^{2} g^{2}|u|^{2}+\frac{g^{2}}{\eta}|u|^{2}\right\} d x \\
& \leq \frac{1}{m}\left(1+\frac{1}{\eta}\right) \int_{\mathbb{R}^{n}}\left(m+\rho(r)^{2}\right)^{-s}|u|^{2} d x .
\end{aligned}
$$

In view of Schwarz' inequality

$$
\langle A \vec{\xi}, \overrightarrow{\vec{\eta}}\rangle^{2} \leq\langle A \vec{\xi}, \overrightarrow{\vec{\xi}}\rangle\langle A \vec{\eta}, \overrightarrow{\vec{\eta}}\rangle \quad\left(\vec{\xi}, \vec{\eta} \in \mathbb{C}^{n}\right)
$$

the estimates (3.7) and (3.8) give

$$
\begin{aligned}
I_{4}+I_{5} & =\int_{\mathbb{R}^{n}}\left\{f_{R}^{2}|\langle A \nabla g, D u\rangle|^{2}+\eta f_{R}^{2} g^{2}\left|\left\langle A \nabla f_{R}, D u\right\rangle\right|^{2}\right\} d x \\
& \leq \int_{\mathbb{R}^{n}} f_{R}^{2}\langle A D u, \overline{D u}\rangle\left[\langle A \nabla g, \nabla g\rangle+\eta g^{2}\left\langle A \nabla f_{R}, \nabla f_{R}\right\rangle\right] d x \\
& \leq\left[\frac{(s+1)^{2}}{m}+\eta C_{4}\right] \int_{\mathbb{R}^{n}} f_{R}^{2}\left(m+\rho(r)^{2}\right)^{-s-1}\langle A D u, \overline{D u}\rangle d x
\end{aligned}
$$

for $\eta, m>0$. Now, we obtain from (3.3), (3.4), (3.9), (3.10), and (3.11) that 


$$
\begin{gathered}
{\left[1-C_{0} C_{3} \eta^{\alpha / 2}-\frac{(s+1)^{2}}{m}-C_{4} \eta\right] \times \int_{\boldsymbol{R}^{n}} f_{R}^{2}\left(m+\rho(r)^{2}\right)^{-s-1}\langle A D u, \overline{D u}\rangle d x} \\
\leq\left\{C_{1}+\frac{C_{2}^{\prime}}{m}+C_{0} \eta^{\alpha / 2}\left[2 C_{3}\left(\frac{s+1}{m}\right)^{2}+\frac{2 C_{3} C_{4}}{m}+1+\frac{C(\eta)}{m \eta^{2}}\right]\right. \\
\left.+\frac{1}{m}\left(1+\frac{1}{\eta}\right)\right\} \int_{\boldsymbol{R}^{n}}\left(m+\rho(r)^{2}\right)^{-s}|u|^{2} d x
\end{gathered}
$$

which shows that for any given $C_{1}^{\prime}>C_{1}$ one can find a sufficiently small $\eta>0$ and a sufficiently large $m_{0}$ so that

$$
\begin{aligned}
& \int_{\boldsymbol{R}^{n}} f_{R}^{2}\left(m_{0}+\rho(r)^{2}\right)^{-s-1}\langle A D u, \overline{D u}\rangle^{2} d x \\
& \leq C_{1}^{\prime} \int_{\mathbb{R}^{n}}\left(m_{0}+\rho(r)^{2}\right)^{-s}|u|^{2} d x .
\end{aligned}
$$

The condition (A.2) implies

$$
f_{R}(x)=\varphi(\rho(r)-R+1) \rightarrow 1 \quad(R \rightarrow \infty)
$$

which, $u \in L_{-s, \rho}^{2}$ and (3.12) give Lemma 3.2 by means of Fatou's lemma.

Q.E.D.

Proof of Theorem 2.1. Let $u \in L_{-s, \rho}^{2} \cap H_{2, l o c}$ be a solution of $L u=z u$ such that $|\operatorname{Im} z|>2 s \sqrt{C_{1}}$. We shall show $u=0$ below. In view of (2.1) there exists a positive number $C_{1}^{\prime}>C_{1}$ such that

$$
\operatorname{Im} z \mid>2 s \sqrt{C_{1}^{\prime}}
$$

Choose a sufficiently large number $m_{0}$ as in Lemma 3.2. Lemma 3.1 is used again with

$$
f=f_{R}=\varphi\left(\frac{\sqrt{m_{0}+\rho(r)^{2}}}{R}\right), \quad g=\left(m_{0}+\rho(r)^{2}\right)^{-s / 2}
$$

( $\varphi$ is the function in the proof of Lemma 3.2) and gives, by taking the imaginary part of the integral, 


$$
\begin{aligned}
& (\operatorname{Im} z) \int_{\mathbb{R}^{n}} f_{R}^{2} g^{2}|u|^{2} d x=\operatorname{Im} \int f_{R}^{2} g^{2}(L u) \bar{u} d x \\
& \left.\quad=2 \int_{\mathbb{R}^{n}} f_{R} g\left\{f_{R} \operatorname{Im}[\langle A \nabla g, D u\rangle \bar{u}]+g \operatorname{Im}\left\langle A \nabla f_{R}, D u\right\rangle \bar{u}\right]\right\} d x
\end{aligned}
$$

We have

$$
\begin{aligned}
& \left\langle A \nabla f_{R}, \nabla f_{R}\right\rangle \\
= & \langle A \hat{x}, \hat{x}\rangle \rho^{\prime}(r)^{2} \rho(r)^{2}\left(m_{0}+\rho(r)^{2}\right)^{-1} \varphi^{\prime}\left(\sqrt{m_{0}+\rho(r)^{2}} / R\right)^{2} / R^{2} \\
\leq & R^{-2} \varphi^{\prime}\left(\sqrt{m_{0}+\rho(r)^{2}} / R\right)^{2}
\end{aligned}
$$

and

$$
\begin{aligned}
\langle A \nabla g, \nabla g\rangle & =\langle A \hat{x}, \hat{x}\rangle s^{2} \rho^{\prime}(r)^{2} \rho(r)^{2}\left(m_{0}+\rho(r)^{2}\right)^{-s-2} \\
& \leq s^{2}\left(m_{0}+\rho(r)^{2}\right)^{-s-1}
\end{aligned}
$$

It follows from (3.14), (3.15), (3.16) and Schwarz' inequality that

$$
\begin{aligned}
& |\operatorname{Im} z| \int_{\mathbb{R}^{n}} f_{R}^{2}\left(m_{0}+\rho(r)^{2}\right)^{-s}|u|^{2} d x \\
& \leq 2\left[\int_{\mathbb{R}^{n}}\left(m_{0}+\rho(r)^{2}\right)^{-s-1}\langle A D u, \overline{D u}\rangle d x\right]^{1 / 2} \\
& \quad \times\left[s\left(\int_{\mathbb{R}^{n}}\left(m_{0}+\rho(r)^{2}\right)^{-s}|u|^{2} d x\right)^{1 / 2}\right. \\
& \left.+\left(\int_{\mathbb{R}^{n}}\left(m_{0}+\rho(r)^{2}\right)^{-s+1} R^{-2} \varphi^{\prime}\left(\sqrt{m_{0}+\rho(r)^{2}} / R\right)^{2}|u|^{2} d x\right)^{1 / 2}\right] .
\end{aligned}
$$

Since the support of $\varphi^{\prime}(t)$ is included in the closed interval [1,2] and $\left|\varphi^{\prime}(t)\right|^{2} \leq C_{4}$, the last integral in (3.17) is estimated by

$$
4 C_{4} \int_{\mathbb{R}<\sqrt{m_{0}+\rho^{2}}<2 R}\left(m_{0}+\rho(r)\right)^{-s}|u|^{2} d x,
$$

which converges to 0 as $R \rightarrow \infty$ by means of $(\mathrm{A}-2)$ and $u \in L_{-s . \rho}^{2}$. Thus, letting $R$ tend to infinity in (3.17), we have 


$$
\begin{aligned}
|\operatorname{Im} z| \int_{\mathbb{R}^{n}}\left(m_{0}+\rho(r)^{2}\right)^{-s}|u|^{2} d x \\
\leq 2 s\left[\int_{\mathbb{R}^{n}}\left(m_{0}+\rho(r)^{2}\right)^{-s-1}\langle A D u, \overline{D u}\rangle d x\right]^{1 / 2} \\
\times\left[\int_{\mathbb{R}^{n}}\left(m_{0}+\rho(r)^{2}\right)^{-s}|u|^{2} d x\right]^{1 / 2}
\end{aligned}
$$

and, by Lemma 3.2

$$
\left(|\operatorname{Im} z|-2 s \sqrt{C_{1}^{\prime}}\right) \int_{\mathbb{R}^{n}}\left(m_{0}+\rho(r)^{2}\right)^{-s}|u|^{2} d x \leq 0,
$$

which and (3.13) show $u=0$.

Q.E.D.

Remark 3.3. In the above proof the condition

$$
V_{1} \in Q_{\alpha, l o c}
$$

is used only to assure $V_{1}|u|^{2} \in L_{l o c}^{1}$ for $u \in H_{2, l o c}$. Therefore, we can adopt in $(\mathrm{A}-4)$

$$
V_{1}|u|^{2} \in L_{l o c}^{1} \quad \text { for any } \quad u \in H_{2, l o c}
$$

instead of (3.18).

\section{$\S 4$. Counterexamples}

In this section we show examples of $L$ with a non-trivial solution $u(x) \in L_{-s, \rho}^{2} \cap C^{\infty}$ of

$$
L u=z u
$$

for $|\operatorname{Im} z|<2 s \sqrt{C_{1}}$.

The following example in $\mathbb{R}^{1}$ is simple.

Example 4.1. Let $L=-\frac{d^{2}}{d x^{2}}-x^{2}$ and $u(x)=\exp \left(-i x^{2} / 2\right)$.

Then $u(x)$ satisfies 


$$
\begin{aligned}
& L u=-u^{\prime \prime}-x^{2} u=i u, \\
& u \in L_{-s}^{2} \cap C^{\infty} \text { for any } s>\frac{1}{2} .
\end{aligned}
$$

This is an example which gives $C_{1}=1, \operatorname{Im} z=1$ and

$$
|\operatorname{Im} z|-2 s \sqrt{C_{1}}=1-2 s<0
$$

for any $s>1 / 2$.

The following example is a generalization of the above.

Example 4.2. Let $t<\frac{n}{4}, \delta \neq 0$ and put

$$
u(x)=\frac{\exp \left(-i \delta r^{2} / 2\right)}{\left(1+r^{2}\right)^{t}} \quad\left(x \in \mathbb{R}^{n}, r=|x|\right)
$$

Then, $u(x)$ satisfies

$$
u \in L_{-s}^{2} \cap C^{\infty}
$$

for any real $s$ such that

$$
s>\frac{n-4 t}{2}
$$

and

$$
L u=\left[-\sum_{j=1}^{n}\left(\frac{\partial}{\partial x_{j}}+i x_{j} g(r)\right)^{2}+V_{1}(x)+V_{2}(x)\right] u=i \delta(n-4 t) u,
$$

where

$$
\begin{aligned}
& g(r)=4 \delta t r^{-n}\left(1+r^{2}\right)^{2 t} \int_{0}^{r} \frac{s^{n-1}}{\left(1+s^{2}\right)^{2 t+1}} d s, \\
& V_{1}(x)=-\delta^{2} r^{2}+2 \delta g r^{2}-g^{2} r^{2}
\end{aligned}
$$




$$
V_{2}(x)=\frac{f^{\prime \prime}}{f}+\frac{(n-1) f^{\prime}}{r f}
$$

where

$$
f(r)=\left(1+r^{2}\right)^{-t}
$$

The identity (4.3) can be shown by a straightforward calculation. We should remark here that we choose $g(r)$ as a solution of

$$
r g^{\prime}+(n-4 t) g+\frac{4 t}{1+r^{2}} g=\frac{4 \delta t}{1+r^{2}} .
$$

One can check immediately that $V_{2}(x) \in C^{x}\left(\boldsymbol{R}^{n}\right)$,

$$
V_{2}(x)=O\left(r^{-2}\right) \text { as } r \rightarrow \infty
$$

and $g(|x|) \in C^{1}\left(R^{n}\right)$ satisfy

$$
g(r)=0\left(r^{-\gamma}\right) \text { as } r \rightarrow \infty
$$

for some $\gamma>0$, which yields that for any positive number $C_{1}<\delta^{2}$ there exists a positive number $C_{2}$ such that

$$
V_{1}(x) \geq-C_{1} r^{2}-C_{2}
$$

Thus, it turns out that the assumptions of Theorem 2.1 are satisfied with $z=\delta i(n-4 t)$ except (2.1). In fact, we have

$$
|\operatorname{Im} z|-2 s \sqrt{C_{1}}=|\delta|(n-4 t)-2 s \sqrt{C_{1}}<0
$$

in view of (4.2) and by taking $C_{1}$ sufficiently close to $\delta^{2}$.

The above example implies that for any real number $s>0, C_{1}>0$ and $\varepsilon \neq 0$ such that

$$
|\varepsilon|<2 s \sqrt{C_{1}}
$$

there exist $L$ and $u \neq 0$ satisfying the condition (A), (4.1) and $L u=i \varepsilon u$. 


\section{Acknowledgement}

The second author would like to thank Professors A. M. Hinz, H. Kalf, G. Stolz, J. Weidmann and E. Wienholtz for stimulating conversations. The hospitality at Frankfurt (am Main) University, where a part of this work was done, is gratefully acknowledged. The authors also wish to thank Professors A. Jensen and J. Uchiyama for valuable discussions and advices.

\section{References}

[1] Ikebe, T. and Kato, T., Uniqueness of the self-adjoint extension of singular elliptic differential operators, Arch. Rat. Mech. Anal., 9 (1962), 77-92.

[2] Ikebe, T. and Saitō, Y., Limiting absorption method and absolute continuity for the Schrödinger operator, J. Math. Kyoto Univ., 12 (1972), 513-542.

[3] Jörgens, K., Wesentliche selbstadjungiertheit singulärer elliptischer Differentialoperatoren zweiter ordnung in $C_{0}^{\infty}(G)$, Math. Scand., 15 (1964), 5-17.

[4] Simon, B., Spectrum and continuum eigenfunctions of Schrödinger operators, J. Func. Anal., 42 (1981), 347-355.

[5] Yamada, O., Spectral theory of magnetic Schrödinger operators with exploding potentials, J. Math. Kyoto Univ., 30 (1990), 585-623. 